\title{
Direitos de propriedade, custos de transação e questões regulatórias
}

\author{
Guilherme Canela de Souza Godoi
}

\section{Resumo}

A regulação exercida por agentes estatais sobre diferentes atores, econômicos ou não, insere-se no rol de instituições que foram e são criadas pelas diferentes sociedades para a solução de problemas que não se resolvem com o simples caminhar da história (ou com o livre mercado, se quisermos nos ater à questão econômica).

Neste sentido, estudar regulação implica entender o seu forte relacionamento com a economia institucional. Por isso, a preocupação central deste trabalho é verificar, na literatura, como as discussões acerca dos custos de transação $e$ dos direitos de propriedade, fundadoras da economia institucional, afetam o entendimento de uma questão institucional específica: a regulação estatal, especialmente aquela realizada através de agentes regulatórios singulares.

De acordo com Williamson (1979, p. 233): "The new institutional economics is preoccupied with the origins, incidence, and ramifications of transaction costs. Indeed, if transactions costs are negligible, the organization of economic activity is irrelevant". Para Aguirre (s/d, p. 3): "É a existência de tais custos [de transação] que justifica a necessidade da definição o mais claro possível dos direitos. Desse modo, os direitos de propriedade são vistos pela NEI [nova economia institucional] como a forma de reduzir as incertezas e com isto os custos associados às 
trocas". ${ }^{1}$ Estas duas observações salientam a importância, para a economia institucional, de dois conceitos, custos de transação e direitos de propriedade, que merecem nossa especial atenção no presente texto.

Por estas duas afirmações podemos inferir a clivagem teórica existente entre diferentes tipos de concepção da realidade: uma que enfoque a importância das instituições (salientada pelas duas citações acima, por exemplo), e outra que apenas avalie a importância dos mecanismos de mercado. ${ }^{2}$ Veja-se que, numa realidade de informação completa, com seres humanos absolutamente racionais e maximizadores, sem incertezas e portanto sem custos de transação, conforme os pressupostos da teoria microeconômica não temos a necessidade do estabelecimento de instituições. No entanto, se não temos informação perfeita, se temos seres humanos com uma racionalidade limitada $e$, portanto, se os custos de transação são uma realidade, a necessidade de institucionalização é imperativa, segundo as colaborações de parte dos teóricos. Em assim sendo, os custos de transação e os direitos de propriedade aparecem como conceitos centrais para o entendimento da realidade social, econômica e política.

Nossa intenção é, a partir de uma análise de como a teoria trata estes dois conceitos, identificar possíveis conexões entre eles e as questões regulatórias. Ou seja, como as discussões acerca dos custos de transação e dos direitos de propriedade, fundadoras da economia institucional, afetam o entendimento de uma questão institucional específica: a regulação estatal, especialmente aquela realizada por agentes regulatórios singulares.

Nesse sentido, dividimos nossa exposição da seguinte forma: primeiro, fazemos uma revisão da teoria que discute custos de transação $e$ direitos de propriedade, conferindo especial atenção às questões que, mais adiante, interessarão à discussão da questão regulatória; na seção seguinte, propomos possíveis abordagens de inter-relacionamento entre os problemas levantados pelos teóricos dos custos de transação $e$ dos direitos de propriedade e as questões regulatórias; em seguida, expomos nossas considerações finais.

Jaffe and Louziotis, Jr. (1996, p. 140) vão na mesma direção.

2 Para um aprofundamento das diferenças entre a Economia Institucional e a Teoria Microeconômica clássica, cf. Eggertsson (1990). 


\section{Custos de transação e direitos de propriedade}

Por onde começar? Custos de transação ou direitos de propriedade? O que vem primeiro? De acordo com a exposição de Aguirre, a existência do custos de transação implica a clarificação da idéia de direitos de propriedade. Poderíamos, então, pensar que o nosso ponto de partida seriam os custos de transação. Mas, vejamos a definição de Niehans (1987, p. 676) para a origem dos custos de transação:

Transactions costs arise from the transfer of ownership or, more generally, of property rights.

Para Coase (1937, p. 390-1):

The costs of negotiating and concluding a separate contract for each exchange transaction which takes place on a market must also be taken into account.

Assim, seja de forma explícita para Niehans seja de forma não tão direta para Coase, os custos de transação se dão quando há uma negociação de direitos de propriedade. Em assim sendo, nos parece uma atitude mais logicamente encadeada iniciarmos nossas discussões pelos direitos de propriedade,$^{3}$ ainda que, em vários momentos, não seja tarefa fácil separar as duas questões.

Os direitos de propriedade só fazem sentido num mundo onde exista a sociedade estabelecida, ${ }^{4}$ e este parece ser um consenso entre os autores que tratam do tema. Para Demsetz (1967, p. 347), não podemos falar em direitos de propriedade num mundo de Robinson Crusoe e, portanto:

An owner of property rights possesses the consent of fellowmen to allow him to act in particular ways.

3 Vamos na linha de Bose (2000, p. 6): "The creation and protection of the right to willfully enter into transactions, or the right to decline to do so - in other words, property rights - is of course fundamental". Ou seja, a própria existência da transação está relacionada ao conceito de direitos de propriedade. Este argumento é reforçado pela explanação de Furniss (1978, p. 400-1): "For the public choiceproperty rights school, negotiations can proceed only after the establishment of a legally sanctioned structure of property rights".

4 Para autores como Coleman (1990) todos os direitos são estabelecidos socialmente. 
De acordo com Alchian (1987, p. 1030), "a property right is a socially enforced right to select uses of an economic good". Novamente, a definição está dependente da variável social. Libecap (2001, p. 3) fala em direitos de propriedade enquanto instituições sociais e salientam que eles determinam o uso aceito dos recursos:

They are among the most critical social institutions, providing the basis for resource-use decisions and for the assignment of wealth and political power. As such, the property regime profoundly influences both economic performance and income distribution in all economies. Property rights define the accepted array of resource uses, determine who has decision-making authority, and describe who will receive the associated rewards and costs of those decisions.

Tendo deixado claro esta característica de definição social dos direitos de propriedade, é nosso intuito, neste momento, nos atermos mais detalhadamente ao trabalho de Demsetz (1967). Um primeiro ponto a ser considerado está relacionado à redução das incertezas. Para o autor (p. 347) os direitos de propriedade cumprem uma função importante, ao permitirem aos indivíduos formar expectativas ao negociarem com outros indivíduos. Demsetz está especialmente preocupado com a relação entre direitos de propriedade e externalidades. Os efeitos benéficos ou nocivos podem ser chamados externalidades quando o custo de fazer com que estes efeitos influenciem as pessoas interagentes é muito alto (p. 348). Logo, a questão para o autor está em como internalizar estas externalidades. Incentivar esta internalização seria uma das funções dos direitos de propriedade. Para Demsetz, portanto, o surgimento dos direitos de propriedade está diretamente relacionado à emergência de diferentes formas de efeitos benéficos e/ou nocivos (p. 350). ${ }^{5}$ Após a explicação de como surgem os direitos de propriedade o autor irá se concentrar na tipificação dos diferentes tipos de direitos, a saber: propriedade comunal, propriedade privada e propriedade esta-

5 O autor utiliza vários exemplos para confirmar suas hipóteses. Para corroborar, especificamente, a questão do surgimento dos direitos de propriedade ele fará uma longa digressão sobre como os indígenas que tinham interesse no comércio de peles procuraram estabelecer direitos de propriedade sobre este comércio quando se sentiram ameaçados por caçadores. 
tal. ${ }^{6}$ A análise do que acontece (ou acontecia) numa situação de propriedade comunal mostra a tendência natural em se passar para a propriedade privada: "Communal property results in great externalities" (p. 355). A conclusão do autor é que os custos de manutenção dos interesses de um determinado proprietário diminuem muito num sistema de propriedade privada, especialmente porque a negociação, quando o proprietário sente que seus interesses são atingidos, é mais simples:

What would be a simple negotiation between two persons under a private property arrangement turns out to be a rather complex negotiation between the farmer and everyone else. (p. 357)

Assim, o estabelecimento dos direitos de propriedade privada, como já exposto por Aguirre, está relacionado à diminuição de diversos custos de transação, já que para Demsetz o resultado da manutenção de propriedades comunais seria o estabelecimento de uma série de contratos entre os diferentes proprietários para que cada um tivesse seus interesses assegurados, aumentando, em muito, os custos. Mais do que isso, para o autor a propriedade comunal é um desincentivo à produção. Para explicitar seu ponto ele cita o exemplo da propriedade intelectual: segundo ele haveria um desestímulo ao avanço das idéias se a propriedade das mesmas fosse comunal, ou seja, se um sistema de patentes não existisse (p. 359).

Alchian (1987) está preocupado com a explanação das diferentes características dos direitos de propriedade. Para ele os direitos de propriedade são socialmente determinados; podem ser alienados, desde que haja uma troca por direitos semelhantes; sua força é medida "pela probabilidade e custos de enforcement", o que, por sua vez, depende do governo, das ações sociais informais e das normas éticas e morais (p. 1031). Para o autor a produção numa sociedade onde o conhecimento é difuso só funcionará de maneira adequada com a existência dos direitos de propriedade; há, portanto, uma relação direta com a diminuição de incertezas (a qual, de seu lado, depende fortemente da garantia dos contratos, na qual o Estado tem um papel preponderante $)^{7}$ e a conseqüente diminuição de custos de transação:

6 Esta discussão também está em Eggertsson, op. cit., p. 36-7 e Jaffe e Louziotis, Jr., op. cit., p. 141.

7 Para um aprofundamento desta perspectiva de garantia dos contratos, cf. Eggertsson, op. cit., p. 45 e ss. 
For the decentralized coordination of productive specialization to work well, according to the well known principles of comparative advantage, in a society with diffuse knowledge, productive resources and products tradable at mutually agreeable prices at low costs of negotiating reliable contractual transactions. That system's ability to coordinate diffused information results in increased availability of more highly valued goods as well as of those becoming less costly to produce. (p. 1031)

Outro ponto de interesse para o autor é a discussão acerca dos recursos que não contam com direitos de propriedade definidos: ar, água, radiação eletromagnética, os bens públicos em geral. Para ele o controle, aqui, passa a ser inteiramente governamental e social, com os diferentes problemas advindos deste tipo de controle.

Eggertsson (1990) irá tratar dos direitos de propriedade na linha do pensamento de Alchian, ressaltando, não obstante, a importância dos custos de enforcement na definição dos direitos de propriedade. A inexistência de mecanismos efetivos de enforcement pode até mesmo inviabilizar determinado conjunto de transações, dado o alto custo das mesmas quando os direitos de propriedade não estão garantidos:

In areas where the state does not help to enforce contracts or even outright prohibits possession and exchange (e.g., of heroin), high transaction costs usually limit or even prevent exchange.

(p. 35)

$\mathrm{O}$ autor enfatiza que os custos para o enforcement dos direitos de propriedade estão relacionados com a existência de normas sociais convergentes com o que o Estado almeja. Do contrário os mesmos podem ser tão altos para a autoridade pública que sus diretrizes podem não ser cumpridas e estruturas de direito paralelas podem ser estabelecidas; é o que ocorre, na visão do autor, com a Máfia (p. 36). De qualquer sorte, o papel do Estado, para Eggertsson, é fundamental quando o tema é direitos de propriedade, não só pelo seu poder enquanto agente do enforcement, mas pelas suas diferentes características interventoras, seja como um agente "atenuante" de direitos seja como o distribuidor inicial dos mesmos (p. 39-40). Por fim, os direitos de propriedade são tão centrais para o sistema econômico que mudanças nos mesmos podem causar importantes alterações no sistema: 
The property rights approach draws attention to the fact that subtle changes in the content of property rights can change the macroperformance of an economic system and lead to economic growth or stagnation. (p. 40)

Jaffe e Louziotis, Jr. (1986) têm por objetivo principal estabelecer a relação entre direitos de propriedade fortes e eficiência econômica. Para tanto, analisam que, apesar dos mais diferentes avanços conquistados pelas sociedades modernas (especialmente na área de tecnologia), a incerteza permanece e, em muitos sentidos, é ainda maior. Por isso uma maior quantidade de informações faz-se necessária para a diminuição do risco associado aos diferentes investimentos. Como para eles "os direitos de propriedade influenciam o risco e o retorno" (p. 155), a análise deste conceito é central:

The reason strong private property rights result in higher level of societal wealth via increased economic efficiency is that strong private property can reduce the risk associated with receiving future returns that the property can potentially generate. Alternatively, this relationship obtains because strong property rights create the proper incentives for economic efficiency by increasing the probability that the holder of those rights will receive the future flows that result from those claims. (p. 139)

Os autores passam, então, a analisar as características dos direitos de propriedade, assumindo posições bastantes semelhantes àquelas expostas anteriormente. Um ponto a ser ressaltado é a subdivisão dos direitos de propriedade em três tipos de direitos: o direito de uso, o direito de exclusão e o direito de transferência ${ }^{8}$ (p. 139). O direito de exclusão, para os autores, será de especial importância na análise das relações entre custos de transação e direitos de propriedade. Segundo eles, os custos de transação - os quais serão positivos quando os direitos de propriedade não estiverem adequadamente definidos - são reduzidos por aqueles indivíduos que conseguem exercer seu direito de exclusão da maneira mais efetiva:

8 Esta subdivisão também é analisada por Eggertsson, op. cit., p. 34 e ss. Libecap (2001, p. 7), traz uma taxonomia diferente: ele fala em direito de uso, direito de se apropriar dos retornos advindos do bem acerca do qual se detém a propriedade e do direito de mudar a forma, substância e localização do bem. 
The ability to exclude reduces the amount of resources that must be used to protect the rights held. This, in turn, reduces the uncertainty associated with the ownership of those rights. Inability to exclude may also result in a free-rider problem, which will also drive up transaction costs. (p. 141)

Os autores continuam sublinhando que o estabelecimento de instituições está diretamente ligado à questão dos direitos de propriedade e que, a partir da participação de uma terceira parte (o governo), intensifica-se uma importante relação: aquela que se dá entre lei e economia (p. 143). A presença do governo ${ }^{9}$ e sua capacidade de enforcement, não obstante, parecem ser a chave do problema da força maior ou menor dos direitos de propriedade: instituições diretamente relacionadas aos diferentes tipos de governos (democracia, por exemplo) estão, historicamente, associadas a uma maior ou menor garantia crível dos direitos de propriedade (p. 144). Os autores mostram, através de uma série de estudos empíricos, que as sociedades mais bem sucedidas instituem os direitos de propriedade como meio de reduzir o risco e aumentar a riqueza daquela sociedade (p. 152).

Libecap (2001) discute a questão dos direitos de propriedade sob duas óticas intimamente conectadas à nova economia institucional: a dos custos de transação e a da mudança institucional. Assim como os autores anteriores, ele explicita - $e$ vai mais além, ao intensificar a análise - as conexões entre direitos de propriedade e custos de transação. Isto certamente ficará mais claro a partir da exposição de algumas teorias acerca destes custos. Quanto à mudança institucional, abdicaremos de aprofundar esta discussão neste momento, visto que esta é uma importante linha de pesquisa na área, inclusive na relação com o problema da regulação, porém vai além do escopo do presente trabalho.

O autor, portanto, está interessado em evidenciar que mudanças nos direitos de propriedade redefinem posições dentro da sociedade ao modificar a configuração da distribuição de poder (político, por exem-

9 O governo é um ator presente em quaisquer análises referentes aos direitos de propriedade, entretanto, Furniss, op. cit., critica a escola dos direitos de propriedade exatamente pelo fato da mesma não apresentar uma discussão adequada acerca da Teoria do Estado e, por isso mesmo "the political implications of public choice-property rights school are cloudy at best" (Ibidem, p. 409). 
plo) e de riquezas no seio da mesma. Este tipo de modificação, a qual se dá, importante frisar, num ambiente de incerteza e de dificuldades de mensuração, está associada a uma série de outros problemas: as partes que possivelmente perderão com a mudança reagirão, tornando necessária uma negociação complexa (p. 4). O encaminhamento sugerido pelo texto é de que a eficiência econômica conquistada com a definição dos direitos de propriedade é algo mais complexo do que a teoria neoclássica teria sugerido. Este fato se deve, em muito, à existência dos custos de transação:

without consideration of transaction costs, it is hard to explain North's [...] observation that property rights institutions that promote efficient resource use are the exception rather than the norm. (p. 4-5)

As diferentes maneiras como os direitos de propriedade são assegurados aos distintos atores componentes de uma sociedade (indivíduos, grupos, Estado) implicam em custos de transações maiores $e$ menores "para a tomada de decisão e para o uso dos recursos". Ou seja, a forma como os direitos estão estruturados tem influência na eficiência do sistema econômico (p. 6). Em assim sendo, as características dos direitos de propriedade (como o sistema de enforcement, por exemplo) são elementos fundamentais na determinação do quão eficazes estes direitos serão. Neste ponto, concordando com autores anteriormente comentados, o autor ratifica a importância do Estado, especialmente na passagem de um sistema de direitos de propriedade sustentado por garantias informais para um sustentado por garantias formais. Mas o Estado, como já apontado num outro momento, também pode ser um complicador do sistema: o Estado pode aumentar as incertezas, pode modificar as expectativas dos agentes - por exemplo, ao modificar de maneira abrupta os direitos de propriedade, gerando, assim, uma menor eficiência econômica (p. 6-7):

If widespread in a society, attenuation of property rights can result in lower economic performance, diminished wealth, and fewer economic opportunities for its members. (p. 7)

Com isso, o autor enfatiza que os acordos que conduzem ao estabelecimento ou a modificações nos direitos de propriedade devem ser analisados sob a ótica dos custos de transação, observando-se o 
tipo de informação disponível, o nível de homogeneidade dos atores envolvidos. Tudo isto pode "explicar a existência de formas ineficientes de direitos de propriedade nas diferentes sociedade e nos diferentes momentos históricos" (p. 24).

Conforme pudemos constatar nos diferentes autores acima expostos, há uma intima relação entre direitos de propriedade e custos de transação. Seja porque a existência dos direitos de propriedade faz sentido ao falarmos da possibilidade de transacioná-los e, dado que eles não são completos, há custos nesta transação, seja porque a clara definição de direitos de propriedade traduz-se numa forma de diminuição destes custos de transação. Mas o que são estes custos? Como eles são gerados? O que pensam diferentes autores sobre os mesmos?

Um trabalho seminal sobre a economia dos custos de transação - ainda que quando da sua publicação os termos não tenham sido colocados desta forma - é o de Coase (1937). O autor está buscando responder a uma questão que lhe pareceu central então: por que num sistema de mecanismo de preços - o qual, supõe-se, funciona no mercado e não exige, a princípio, uma forma de organização - as organizações, no caso dele especificamente as firmas, surgem? (p. 388). Em outras palavras, por que é lucrativo estabelecer uma firma? (p. 390). Segundo ele, a resposta a esta questão está dada pela existência de custos em se utilizar o mecanismos de preços. Estes custos advêm de algumas características do próprio mecanismo. Dentre elas Coase está preocupado, especialmente, com o custo de obtenção de informação (é preciso identificar "quais são os preços relevantes") e com o custo de se firmar contratos para cada transação realizada (p. 390-1). As firmas não reduziriam totalmente estes custos, mas os diminuiriam substantivamente:

We may sum up this section of the argument by saying that the operation of a market costs something and by forming an organization and allowing some authority (an "entrepreneur") to direct the resources certain marketing costs are saved [...] A firm, therefore, consists of the system of relationships which comes into existence when the direction of resources is dependent on an entrepreneur. (p. 392-3).

Desta percepção é importante termos claro que as organizações, no caso as firmas, reduzem os custos de transação que existem numa 
situação de mercado regido pelo mecanismo de preços. Não obstante, os custos continuam existindo, visto que os problemas de informação não são totalmente resolvidos e os custos de estabelecimento de contratos (agora em menor número) também não são totalmente suprimidos. ${ }^{10}$ Dito isto, uma questão que aparece por dedução lógica é posta pelo autor: se é verdadeiro que as firmas reduzem os custos advindos do mecanismo de preços, por que o mercado ainda existe? A resposta, para ele, está relacionada a três constatações: os custos de organizar transações adicionais dentro da firma podem crescer; com o crescimento das transações o entrepreneur falha no sentido de fazer o melhor uso dos fatores de produção; parte dos fatores de produção teriam seu preço de oferta aumentado em função do crescimento da firma (p. 394-5).

O trabalho empírico de Demsetz (1968) analisando os custos de transação no mercado de ações de New York também traz algumas questões relevantes para a discussão do conceito. Um primeiro ponto levantado pelo autor, o qual queremos sublinhar aqui, é que, na ausência de custos de transação, situações de externalidades ou de monopólios podem ser economicamente eficientes (p. 33). Esta constatação evidencia a importância dos custos de transação para as questões institucionais, pois, de um lado, como vimos, a existência das externalidades é central no estabelecimento dos direitos de propriedade por exemplo, e, por outro, a questão dos monopólios, como veremos, é central para a discussão regulatória. Num mundo, portanto, onde estas questões fossem irrelevantes para a eficiência econômica não faria sentido tecermos as discussões institucionais que estamos tecendo no presente trabalho. Na linha de uma definição já colocada anteriormente, o autor define os custos de transação como "os custos de troca de títulos de propriedade" (p. 35). Estes custos podem ser custos de informação acerca de como funciona um determinado mercado, ou até mesmo custos de ligações telefônicas ou de leitura de jornais, como coloca o autor (p. 35). O objetivo do trabalho de Demsetz é verificar em que medida os custos de transação são afetados pela escala de negócios. Chega à conclusão, após a análise empírica, que o custo de negociar um título diminui na medida em que a atividade de negociar aquele título aumenta.

10 Aliás, Coase, no decorrer do artigo, preocupa-se com a relação entre o tamanho da firma e seus custos internos de organização (Coase, op. cit., p. 396-7). 
Tanto o trabalho de Coase como o de Demsetz ressaltam a importância da informação para a existência dos custos de informação. Num mundo onde a informação não é perfeita, obtê-la é custoso. ${ }^{11}$ Nesse sentido, a apresentação de algumas discussões sobre a questão da informação são pertinentes.

Alchian (1950) procura verificar as relações entre a incerteza e a teoria econômica. Sua proposta inicial é a adoção de axiomas distintos, relativamente aos considerados até aquele momento, na construção da teoria econômica: informação incompleta e previsão incerta. A adoção de tais axiomas implica, necessariamente, a revisão no conceito de comportamento individual assumido pela teoria econômica neoclássica:

Adaptive, imitative, and trial-and-error behavior in the pursuit of "positive profits" is utilized rather than its sharp contrast, the pursuit of "maximized profits". (p. 211)

Segundo Alchian a incerteza, central na transformação do entendimento acerca da ação individual, advém de duas fontes: "previsão imperfeita e a inabilidade dos indivíduos em resolver problemas complexos, que contenham uma série de variáveis, mesmo quando um ótimo seja passível de definição" (p. 212). Dada a incerteza, de acordo com o autor, não podemos falar mais num único resultado maximizador, mas sim numa série de resultados potenciais, sendo que o indivíduo deverá optar por um deles. Se a maximização de lucros não é a forma pela qual os indivíduos obtêm sucesso, qual é, então? Alchian discute, na seqüência, o sucesso baseado nos resultados e não na motivação, o papel da sorte e do comportamento adaptativo na obtenção do sucesso. Nos ateremos a este último. Para o autor, "nem o perfeito conhecimento do passado nem completa consciência da situação presente provêm suficiente capacidade de previsão capaz de indicar uma ação maximizadora de lucros". Na tentativa de obter sucesso num mundo de incertezas, portanto, um dos comportamentos adaptativos utilizados pelos indivíduos é a cópia de padrões, ou seja, dada a incerteza há um excelente motivo para a cópia de um comportamento bem sucedido já observa-

11 É importante salientar que Coase, no artigo aqui exposto, está ressaltando que a incerteza, comumente associada à assimetria de informação, não é fator determi nante no estabelecimento das firmas, ele está inclusive indo de encontro àqueles que afirmam isto. Esta, no entanto, é uma questão controversa entre os analistas do trabalho de Coase (Cf. Pessali, p. 14). 
do. (p. 218-9). ${ }^{12}$ Uma segunda forma de comportamento adaptativo se dá através dos erros e acertos, numa espécie de teoria da evolução. Satisfeitas algumas condições, ${ }^{13}$ este processo convergiria para um equilíbrio de maximização de lucros. O importante desta discussão é que a incerteza existe e que ela molda o comportamento individual, tornando mais complexo o alcance dos objetivos econômicos. Logo, a diminuição das incertezas é um anseio do sistema: a introdução de instituições, para uma parte da teoria, é uma forma de reduzi-las.

Uma ilustrativa abordagem acerca do problema da informação (e da incerteza gerada pela assimetria da mesma) é a de Akerlof (1970). Neste artigo, o autor procura, a partir da análise do mercado de carros bons e ruins (lemons nos Estados Unidos) estabelecer as relações entre qualidade dos bens e incerteza. O exemplo do mercado de carros é ilustrativo especialmente pela existência de um mercado de carros novos e usados. Quando se compra um carro no mercado de carros novos há uma desinformação generalizada acerca da qualidade do carro. Todos sabem, no entanto, que há uma probabilidade $(1-\mathrm{p})$ de se estar comprando um carro em boas condições. Não obstante, após a compra de um determinado carro tem lugar uma assimetria de informações entre o dono daquele carro e potenciais compradores do mesmo, já que o proprietário tem informações privilegiadas acerca da qualidade de seu veículo. Assim, o mercado de carros usados acaba generalizando a idéia da venda destes carros por um preço inferior ao dos carros novos, pois, do contrário, haveria um estímulo à venda de carros de má qualidade (lemons), visto que apenas os donos teriam esta informação, para a consecutiva compra de um outro carro no mercado de novos. Destarte, como aponta o autor, os proprietários de carros usados de boa qualidade estão em xeque (p. 489). Este problema aparece nos mais variados mercados e necessita ser solucionado. Para tanto, de acordo com Akerlof, algumas instituições são introduzidas para dirimir os efeitos da incerteza quanto à qualidade. Dentre elas encon-

12 Segundo Alchian (1950, p. 218) a cópia dos padrões se dá por uma série de razões, são elas: the absence of an identifiable criterion for decision-making, the variability of the environment, the multiplicity of factors that call for attention and choice, the uncertainty attaching to all these factors and outcomes, the awareness that superiority relative to one's competitors is crucial, and the nonavailability of a trial-and-error process converging to an optimum position.

13 Para Alchian, (p. 219), estas condições são de difícil consecução. 
tramos: os sistemas de garantias, as marcas de produtos - assim como cadeias de hotéis, restaurantes, perfumaria - e os respectivos compromissos que as empresas assumem ao anunciarem que aquele produto tem aquelas qualidades, as necessárias autorizações para o fornecimento de alguns serviços (licença para exercer a medicina, por exemplo) (p. 499-500). A conclusão do autor reforça suas ponderações ao longo do texto:

[...] the difficulty of distinguishing good quality from bad is inherent in the business world; this may indeed explain many economic institutions and may in fact be one of the more important aspects of uncertainty. (Ibidem, p. 500)

Como já adiantado por Coase, um importante fator na geração de custos de transação é o estabelecimento dos contratos. Este fator passa a ser ainda mais central quando assumimos que os contratos não são completos e, portanto, necessitam ser garantidos por instituições. Está é a suposição de Klein (1980, p. 356):

I start by noting that complete, fully contingent, costlessly enforceable contracts are not possible.

De acordo com o autor, a incompletude dos contratos se deve a dois fatores: a incerteza gera a possibilidade de existirem uma série de problemas futuros que não estão sendo abordados pelas condições contratuais, "sendo que é muito custoso saber e especificar respostas para todos os possíveis problemas". Em segundo, algumas questões específicas são de difícil mensuração $e$, portanto, acabam por não contando com claros mecanismos de enforcement. Há, de acordo com o autor, uma série de custos de transação decorrentes destas incompletudes contratuais. Ele aponta, portanto, que é preciso verificar quais são os melhores desenhos contratuais e quais são os melhores mecanismos de enforcement diante de situações específicas.

Williamson $(1979,1986)$ aparece como um dos mais importantes pensadores da teoria dos custos de transação. Seus trabalhos intensificam e ampliam os estudos iniciados por Coase e explicitam, de maneira clarividente, os pressupostos desta corrente teórica. A primeira preocupação do autor (1979) é buscar elementos de consenso ao redor da questão dos custos de transação e, a partir disto, partir para uma definição mais completa do que sejam estes custos. As questões 
consensuais, ${ }^{14}$ de acordo com o autor, são a de que o oportunismo é um conceito central no estudos dos custos de transação, a de que o processo de informação está estreitamente relacionado a este estudo, $e$ que considerar os custos de transação é considerar as instituições. $\mathrm{O}$ que Williamson (1979, p. 234) mais especificamente pretende é relacionar as características das transações com as estruturas de governança "mais econômicas". Para o autor, um importante ponto de partida na discussão dos custos de transação (assim como muitos dos autores vistos anteriormente) é a questão dos contratos. Por isso ele inicia sua discussão analisando três conjuntos diferentes de contratos: o clássico, cujas principais características são as regras legais, os documentos formais e as transações que se auto-liquidam (p. 237); o neoclássico, o qual, ao contrário do anterior, reconhece os problemas relacionado às incertezas, "a complexidade do mundo, a incompletude dos acordos e que alguns contratos jamais serão alcançados a menos que ambas as partes tenham confiança os mecanismos do acordo" (p. 238); a terceira forma é o contrato relacional, a qual tem como característica principal o enfoque não no acordo inicial, mas em como as relações se desenvolvem ao longo do tempo. Da constatação de que as transações podem ser regidas por diferentes formas contratuais segue-se uma importante definição: "as estruturas de governança - matrizes institucionais nas quais as transações são negociadas e executadas variam conforme a natureza das transações" (p. 239). Como discutido anteriormente, estas estruturas merecem especial atenção do autor. A natureza das transações está relacionada a algumas características específicas, a saber:

[...] (1) uncertainty, (2) the frequency with which transactions recur, and (3) the degree to which durable transaction-specific investments are incurred. (p. 239)

Na seqüência o autor fará uma longa discussão sobre a economia da idiossincrasia, ${ }^{15}$ a qual não será por nós analisada neste traba-

14 Apesar de autor falar em questões consensuais há uma série de divergências com relação a estas mesmas questões (Cf. Pessali, op. cit., cap. 4) e à nova economia institucional como um todo (para uma crítica Cf. Pereira, s/d)

15 As transações idiossincráticas ocorrem quando "specific identity of the parties has important cost-bearing consequences". [grifo do autor] (Williamson, 1979, p. 240). 
lho. A partir da análise da economia da idiossincrasia o autor propõe um dos principais argumentos do paper: "estruturas especiais de governança suplantam trocas contratuais clássicas do mercado quando os valores de transações específicas são altos" (p. 244). A partir dos tipos de contrato e dos tipos de transação (mais específicas ou não) o autor identifica os diferentes tipos de governança: governança de mercado (contrato clássico e transações não específicas); governança trilateral (contrato neoclássico e transações semi-específicas e específicas); governança de transações específicas (contrato relacional), as quais se dividem em duas: a governança bilateral e a governança unificada (a qual tem a ver com a organização interna as organizações). Os diferentes tipos de governança e as características das transações têm implicações diferenciadas para diferentes mercados (como o de trabalho, por exemplo), o que é analisado por Williamson (p. 255 e ss.). A questão central, portanto, é a de que a economia dos custos de transação está diretamente relacionada aos tipos de contratos que regem as transações, os quais, por sua vez, determinam as diferentes estruturas de governança em cujos arcabouços as transações ocorrem, sendo que essas estruturas também sofrem a influência das três principais características associadas às transações: incerteza, freqüência e nível de especificidade.

Em outro momento, Williamson (1986) explicita, de forma mais contundente, quais são os pressupostos da economia da governança e quais são suas implicações para o estudo das transações. Uma relevante constatação inicial é a de que a economia dos custos de transação compartilha de uma perspectiva multidisplinar, aglutinando aspectos do direito, da economia e das teorias de organização (p. 172). O autor começa por estabelecer os pressupostos e principais características da economia dos custos de transação. O objetivo desta vertente da economia, de acordo com Williamson, é estudar o processo de definição e execução dos contratos de maneira completa (ou seja, ex ante e ex post). Para isso é fundamental dois tipos de definições: uma quanto ao tipo de indivíduo que está sendo considerado e outra quanto às maneiras como este indivíduo age. A primeira pressuposição é de que a racionalidade do indivíduo é limitada, é uma forma de racionalidade semiforte, segundo o autor. Partindo da definição de Simon, segundo a qual os indivíduos são "intendedly rational, but only limitedly so" 16

16 Grifos do autor. 
(p. 173), Williamson sublinha porque os dois componentes da definição são fundamentais para a economia dos custos de transação:

The intended-rationality part of the definition calls for an economizing orientation, and a recognition that competence is limited encourages the study of institutions. (p. 174)

O tipo de motivação assumido pela economia dos custos de transação é a oportunística, diretamente relacionada à incerteza - uma das características já apontadas por Williamson. Ou seja, o oportunismo está relacionado à existência de informação incompleta e, especialmente, ao cálculo de esforços para "mislead, disguise, obfuscate, ou confuse" (p. 175). Dentro desta perspectiva principais e terceiras partes têm um desafio muito maior. Uma outra característica dos agentes que deve, na visão de Williamson, ser considerada é a dignidade ( $\mathrm{p}$. 177). Não obstante a importância desta discussão, não mergulharemos nela nesta exposição.

As três dimensões das transações (especificidade dos ativos, incerteza e freqüência) voltam a ser preocupações do autor aqui (p. 179 e ss.). Como no artigo anterior ele enfatiza a relação da especificidade do ativo com o tipo de organização e de salvaguardas que são consideradas; as estruturas de governança variam para responder adequadamente aos distúrbios gerados pelas incertezas; o tamanho dos mercados (freqüência das transações) também terá influência nas diferentes estruturas de governanças, as quais respondem diferentemente a pequenos e grandes mercados. Dados estes pontos de partida o autor analisará algumas implicações para o processo como um todo e para questões específicas (p. 184 e ss.)

Eggertsson (1990) retoma muitas das questões acima discutidas, porém com uma visão mais abrangente dos custos de transação, visto que está retomando o argumento de uma série de autores. A preocupação com a relação entre custos de transação e alocação de recursos e com as questões contratuais, especialmente com o arranjo dos contratos ex ante e o monitoramento ex post, é primordial (p. 14). Outro ponto de inter-relação com os autores discutidos anteriormente é a questão da informação incompleta. Para o autor os custos de transação foram introduzidos tardiamente na discussão econômica, dado que se considerava a informação como completa e estes custos estão associados, na visão dele, à aquisição de informações. Para Eggertsson 
poderíamos levantar várias atividades que, num mundo de informação incompleta, geram custos de transação (busca de informações sobre preços, qualidade e trabalho, além dos comportamentos de vendedores e compradores; a tentativa de identificação da posição de compradores e vendedores no sistema de preços; a produção de contratos; o monitoramento de contratos; o enforcement de contratos; a proteção dos direitos de propriedade) (p. 15). Para Eggertsson, o Estado entra como um ator central na redução dos custos de transação:

When a state introduces and enforces the rule of law in a lawless area, it thereby lowers transaction costs and stimulates trade. [...] Historically, the state has lowered transaction costs by establishing and maintaining stable money, because rapid inflation, particularly variable and unpredictable inflation, increases the cost of transacting. (p. 16)

Na seqüência o autor aborda questões já apresentadas aqui: custos de transação e eficiência, problemas de medida e qualidade dos bens, para então entrar na relação entre custos de transação e equilíbrio, o que foge ao escopo do presente trabalho.

Nosso intuito foi o de que as discussões sobre custos de transação e direitos de propriedade tenham apresentado algumas das principais questões que as linhas de pesquisas nestas duas áreas têm levantado. A seguir, relacionaremos essas questões com o problema da regulação.

\section{Direitos de propriedade, custos de transação e regulação}

A intenção da presente seção é levantar alguns inter-relacionamentos entre os conceitos de direitos de propriedade e custos de transação e a questão regulatória, com o objetivo de estabelecer possibilidades futuras de aprofundamento. Vai além dos limites da presente discussão intensificar as discussões que terão início aqui. Esperamos no entanto que os indicativos abram caminhos para pesquisas futuras. A exposição, nesta seção, procurará seguir a mesma ordem da exposição na seção anterior.

Na definição de regulação dada por Breyer e MacAvoy (1987, p. 128-9), encontramos que: 
Regulation, particularly in the United States, consists of governmental actions to control price, sale and production decisions of firms in an avowed effort to prevent private decision-making that would take inadequate account of the "public interest". The Federal Congress established the first national regulatory agency (The Interstate Commerce Commission) in 1887 [...] regulation is required to overcome one or more 'defects' that prevent corporation's form operating according to the norm.

Os "defeitos" de que falam Breyer e MacAvoy (1987) são a presença de poder monopolístico, a questão dos spillover costs, a informação inadequada, dentre outros.

De acordo com Melo (2000) a teoria normativa da regulação identifica falhas de mercado que devem ser corrigidas com o estabelecimento do aparato regulatório:

As falhas de mercado identificadas pela teoria são: bens públicos, falhas de competição (monopólios), externalidades, mercados incompletos e informação assimétrica. (p. 13)

Para Levy e Spiller (1994) a regulação pode ser entendida como um "contracting problem".

Estas três visões de regulação sintetizam questões que foram abordadas por todos os autores analisados quando o foco de nossa discussão eram os direitos de propriedade e os custos de transação. Nesse sentido, a relevância conferida às externalidades pelos teóricos dos direitos de propriedade e à assimetria de informações e à questão dos contratos pelos teóricos dos custos de transação está diretamente relacionada aos problemas que afligem os teóricos da regulação. Demsetz (1968), coloca que na ausência de custos de transação as externalidades e os monopólios não seriam um problema para o sistema e, portanto, não seria necessário haver regulação. ${ }^{17}$ Como os autores da teoria da regulação acima citados colocaram, estas duas questões são centrais para o estabelecimento de marcos regulatórios, daí a conclusão da estreita relação entre custos de transação e regulação; como era de se esperar, aliás, visto que a regulação nada mais é do que uma

17 Estache e Martimort (1997, p. 5) vão na mesma direção: In a world of perfect information, decreasing technological returns and numerous atomistic agents, there is no need for a government. 
questão institucional. Caminhemos, então, para alguns comentários específicos.

Na definição de Libecap (2001) dos direitos de propriedade aparece que estes estão relacionados a quem tem a autoridade de decidir. Nesse sentido, os direitos de propriedade podem configurar-se num importante instrumental teórico na discussão da regulação estatal sobre "ativos" que são de propriedade pública e cuja exploração é transferida, pelo Estado, a um ente privado. Aqui, também é de especial importância a divisão dos direitos de propriedades em três tipos, conforme acima discutido. Sendo o Estado quem tem o direito, por exemplo, sobre o espectro eletromagnético, ele tem o direito de transferir a exploração da concessão a um terceiro. Quais são as implicações disto pela teoria dos direitos de propriedade? O Estado passa de proprietário do direito a regulador, o que isto significa?

A questão dos direitos de propriedade como direitos de exclusão também é de ampla importância para as questões regulatórias. No campo da regulação de serviços públicos, por exemplo, os direitos de exclusão precisam ser claramente definidos pelo órgão regulador, pois do contrário podem gerar fortes conflitos de interesses entre as empresas competidoras do sistema. $\mathrm{O}$ caso das empresas concessionárias de telefonia e eletricidade é típico: privatizadas as empresas (de um detentor de direitos de propriedade - o Estado - passamos a ter vários), as empresas de eletricidade reclamaram pelo direito de cobrar pela passagem dos cabos telefônicos por seus postes, o que antes era gratuito. As agências reguladoras das duas áreas (elétrica e telecomunicações) tiveram que intervir para que um preço que não alterasse em muito a composição dos preços das tarifas telefônicas pudesse ser estabelecido. A este tipo de questão também podem ser aplicadas às discussões referentes ao problema dos direitos de propriedade comunais acima descritos. Estes direitos também são importantes na análise da utilização comum de bens públicos como água, meio ambiente, estradas, etc., os quais também contam com regulação efetiva por parte do Estado. O caso da água é ilustrativo: a agência reguladora de águas foi compelida a definir muito claramente os direitos de propriedade sobre as bacias hidrográficas para impedir que usinas hidrelétricas de propriedade do setor privado impedissem o fornecimento de água para as regiões circunvizinhas. Os próprios detentores de direitos de propriedade recorrem às agências para que estas garantam o seu direito de exclusão. $\mathrm{O}$ 
exemplo das empresas de radiodifusão é importante: com a multiplicação de rádios piratas, que invadem o espectro eletromagnético prédefinido pelas concessões governamentais, muitas concessionárias têm o seu direito de exclusão violado e, portanto, recorrem à Agência de Telecomunicações para que esta retire do ar a rádio que está violando o direito.

O conceito de direitos atenuados está também amplamente conectado à questão da regulação. Vejamos, novamente, o caso da telefonia: as empresas que compraram o sistema Telebrás e portanto adquiriram direitos de propriedade sobre as diversas empresas do sistema foram obrigadas, pelo órgão regulador, a aceitar uma série de atenuações dos seus direitos. Por exemplo, não podiam atuar em todas as áreas do país dentro de um certo período de tempo, operadoras locais tinham que permitir a operadoras de longa distância o uso de suas redes de conexão.

A questão dos contratos é central, tanto do ponto de vista dos direitos de propriedade quanto dos custos de transação. Além de Levy e Spiller (1994), autores como Estache e Martimort (1997) e Melo (2000) também estão preocupados com papel das agências diante de uma situação de contratos incompletos. Para eles são as agências reguladoras que garantem a credibilidade dos contratos, através de uma série de mecanismos, como os que evitam a captura das agências por grupos de interesse e os que mantêm sua independência política, por exemplo. Alchian (1987, p. 1031), como vimos, fala da importância das "transações contratuais confiáveis"; para Eggertsson (1990, p. 46):

The state, by using its police power and the courts, assists private individuals in enforcing legitimate contracts and thus lowers the costs of exchange, particularly when the state uses its power to enforce contracts in a systematic and predictable manner. [grifos do autor]

As agências reguladoras seriam os melhores órgãos burocráticos para alcançar esse enforcement sistemático e previsivel? Jaffe e Louziotis, Jr. (1986, p. 144) falam do risco advindo de uma ação do Estado contra os direitos de propriedade. Agências regulatórias independentes e críveis seriam uma maneira de diminuir este risco político? Estes autores concluem sua análise sobre os direitos de propriedade e a eficiência econômica fazendo cinco proposições, diante das quais podemos le- 
vantar questões a serem desvendadas no âmbito de uma discussão regulatória. (1) As instituições podem ter efeitos dramáticos no uso dos recursos, algumas podem aumentar os custos de transação, outras diminuir (p. 153). Que características garantem políticas regulatórias que diminuam os custos de transação? (2) Comparações de arcabouços institucionais devem explicar diferenças observadas no tratamento dos ativos em diferentes comunidades políticas, sociais e legais (p. 154). A comparação dos marcos regulatórios de diferentes países confirmaria esta proposição? (3) Na economia global, normas culturais e escolhas políticas podem ser testadas por seus efeitos num amplo espectro de parâmetros (p. 154). Como o sucesso ou fracasso de políticas regulatórias pode ser explicado a partir desta proposição? (4) Pesquisas preliminares mostram que o índice de crédito de um país pode ser determinado por suas escolhas quanto aos direitos políticos, liberdades civis e grau de liberdade econômica (p. 154). Estas características determinam um maior ou menor grau de respeitabilidade das instituições regulatórias também? (5) Se fatores institucionais afetam a eficiência econômica, nós deveríamos ser capazes de estimar preços implícitos para cada instituição. (p. 154) Como poderíamos aplicar isto para as instituições regulatórias?

O trabalho de Coase também acrescenta algumas questões importantes na definição da relevância das agências. Elas poderiam ser entendidas como as firmas de Coase? Ou seja, poderíamos entender o papel das agências como redutoras dos custos de informação e dos custos de transação referentes ao número de contratos necessários? $\mathrm{E}$ mais, a introdução das agências geram custos de transação adicionais? Quando a existência das agências é economicamente ineficiente? ${ }^{18}$

A questão das assimetrias de informação também é relevante para os teóricos da regulação. Para eles há diversos tipos de assimetrias no processo, os quais geram custos adicionais e precisam ser enfrentados pelas agências. Há assimetrias de informação entre os compradores e vendedores na determinação dos preços, devendo a agência intervir, em muitos casos, para que o nível de preços em áreas relevantes - como as utilidades públicas - atendam ao interesse público; há as-

18 Dentro da relação entre regulação e custos de transação, Niehans (1987, p. 678) coloca que: "Regulation, therefore, may be efficient, not because there is an externality, but because regulation may be cheaper than a multilateral contract". 
simetrias de informação entre as firmas reguladas e as agências também no tocante aos preços: desta vez os teóricos apontam que as firmas possuem maiores informações quanto aos seus reais custos, podendo enganar as agências no momento da definição final dos preços; há assimetrias de informação entre consumidores e empresas, na linha das argumentações de Akerlof: assim, por exemplo, como os consumidores não conseguem aferir a qualidade de produtos - como medicamentos - ou de serviços - como aviação -, agências reguladoras devem garantir a qualidade dos bens que estão sendo oferecidos no mercado.

Por fim, as atividades apontadas por Eggertsson como geradoras de custos de transação podem ser um importante foco de discussões regulatórias: (1) A busca de informação sobre a distribuição do preço $e$ qualidade das commodities e do trabalho e a busca por potenciais compradores e vendedores e por informação relevante sobre o comportamento e circunstâncias dos mesmos - quanto a esta questão, tocamos em mais de uma ponto dela no parágrafo anterior; (2) a barganha necessária para encontrar a verdadeira posição de compradores e vendedores quando os preços são endógenos, também já discutido anteriormente; (3) a produção dos contratos: as questões ex ante relativas aos contratos são, como já salientado, de extrema importância para a economia dos custos de transação e também para a questão regulatória - como determinados mecanismos estabelecidos ex ante podem colaborar para o melhor alcance do interesse público em determinados nichos regulatórios?; (4) o monitoramento dos contratos: qual é o papel das agências no momento ex post da definição dos contratos, $e$ qual é o melhor arcabouço regulatório para garantir que os contratos sejam cumpridos?; (5) o enforcement dos contratos e a coleção de danos quando os parceiros falham em observar suas obrigações contratuais: qual é o desing regulatório mais adequado para garantir o enforcement a um custo não elevado?; (6) a proteção dos direitos de propriedade contra terceiros, também já discutido exaustivamente.

Estas são algumas das perspectivas institucionalistas que podem ser utilizadas numa agenda de pesquisa das questóes regulatórias, todas elas necessitando aprofundamento, especialmente para o caso brasileiro. 


\section{Conclusões}

Informação imperfeita, incerteza, externalidades, mercados monopolísticos, problemas na definição e monitoramento dos contratos, problemas na definição dos preços são algumas das questões que certamente compõe um conjunto de interseção entre as teorias dos direitos de propriedade, dos custos de transação e da regulação. Certamente isto se dá porque todas estas são questões institucionais. Não obstante, há uma variedade de tratamentos dispensados pelos pesquisadores que se dedicam à análise dos três tipos de teorias acima mencionadas que podem ser utilizados de forma mais intensa como instrumental para o entendimento dos problemas que compõem este conjunto de interseção.

Procuramos aqui levantar quais possíveis caminhos poderiam ser seguidos se tratássemos a questão regulatória a partir de uma perspectiva dos direitos de propriedade e dos custos de transação.

\section{Referências bibliográficas}

AguirRE, B. Conflitos e instituições: reforma fiscal em uma perspectiva comparada, s/d. Disponível em: < http://www.clacso.edu.ar/ libros/anpocs/aguirre.rtf/>

AKERLOFF, G. The market for "lemons": qualitative uncertainty and the market mechanism. The Quarterly Journal of Economics, 84, p. 488500, 1970.

AlchiAn, A. Uncertainty, evolution, and economic theory. Journal of Political Economy, 58, p. 211-21, 1950.

.; Demsetz, H. Production, information costs, and economic organization.

The American Economic Review, 1972, v. 62, p. 777-95.

Property rights. In: The new palgrave: a dictionary of economics. London: Macmillan Press, 1987, v. 3, p. 1031-4.

ARRUNADA, B. The enforcement of property rights: comparative analyses of institutions reducing transaction costs in real estate.

Bose, G. Institutions and institutional change: a review of conceptual and analytical issues. Prepublication version of institutions and institutional change: a review of conceptual and analytical issues. In: LIMAM (Ed.): Institutional reform in the MENA region, Economic Research Forum, Cairo, 2000. p. 11-44.

BREYER, S.; MACVoY, P. W. Regulation and deregulation. In: The new palgrave: a dictionary of economics. London: Macmillan Press, 1987, v. 4, p. 128-34. 
CoAse, R. The nature of the firm. Economica, v. 4, November, p. 386-405, 1937.

Coleman, J. Foundations of social theory. Cambridge: The Belknap Press of Harvard University Press, 1990.

Demsetz, H. Toward a theory of property rights. American Economic Review, 57 (2), p.347-59, 1967.

The cost of transacting. Quarterly Journal of Economics, v. 82, p. 3353, feb. 1968.

Eggertsson, T. Economic behavior and institutions. Cambridge Surveys of Economic Literature. Cambridge University Press, 1990.

Elster, J. Social norms and economic theory. Journal of Economic Perspectives, 3, p. 99-117, 1989.

Estache, A.; Martimort, D. Transaction costs politics and regulatory institutions. 1997. Mimeografado.

FuRnISS, N. The political implications of the public choice-property rights school. American Political Science Review. 72, p. 399-410, 1978.

HaRT, O. Norms and the theory of the firm. Harvard Institute of Economic Research, Discussion Paper 1923, May 2001.

JAFFE, A. J.; Louziotis JR., D. Property rights and economic efficiency: a survey of institutional factors. Journal of Real Estate Literature, v. 4, n. 2, July, 1986.

JoHnSON, R. N.; LiBECAP, G. D. Contracting problems and regulation: The Case of the Fishery. American Economic Review, 72 (5), p. 1005-22, 1982.

KLEIN, B. Transaction cost determinants of 'unfair' contractual arrangements. The American Economic Review. Borderlines of Law and Economic Theory, v. 70, n. 2, p. 356-62, 1980.

LEVY, B.; SPILLER, P. The institutional foundations of regulatory commitment: a comparative analysis of telecommunications regulation. The Journal of Law, Economics \& Organization, v. 10, n. 2, p. 201-45, 1994.

LiBECAP, G. D.; WigGiNs, S. N. The influence of private contractual failure on regulation: the case of oil field unitization. Journal of Political Economy, 93 (4), p. 690-714, 1985.

. A transaction costs approach to the analysis of property rights. In: Brousseau, E.; Glachant, J. (Eds.). The economics of contracts in prospect and retrospect, April, 2001.

MeLo, M. A. Política regulatória: uma revisão da literatura. In: BIB, Rio de Janeiro, n. 50, p. 7-43, 2000.

NiEHANS, J. Transaction costs. In: The new palgrave: a dictionary of economics. London: Macmillan Press, 1987. v. 4, p. 676-9. 
Pereira, C. B. Notas para uma crítica institucionalista da "nova economia institucional". FEA/USP. Série de Working Papers, 01/009. Disponível em: $<$ http://www.ead.fea.usp/wpapers, s/d/>.

PESSALI, H. F. Teoria dos custos de transação: uma avaliação à luz de diferentes correntes do pensamento econômico. Curitiba, 1998. Dissertação (Mestrado) - UFPR.

PondÉ, J. L.; Fagundes, J.; Possas, M. Custos de transação e políticas de defesa da concorrência. Mimeografado.

WiLLINAMSON, O. E. Transaction-cost economics: the governance of contractual relations. Journal of Law and Economics, 22 (2), p. 233-61, 1979.

The economics of governance: framework and implications. In: LANGLOIS, R. N. (Ed.). Economics as a process: essays in the new institutional economics. Cambridge University Press, 1986, p. 171-202. 